\title{
AN INVESTIGATION OF IRREGULAR CRACK PATH EFFECTS ON FRACTURE MECHANICS PARAMETERS USING A GRAIN MICROSTRUCTURE MESHING TECHNIQUE
}

\author{
A. Mehmanparast*, F. Biglari, C. M. Davies, K. M. Nikbin \\ Mechanical Engineering Department, Imperial College London, Exhibition Road, London, SW7 2AZ
}

\begin{abstract}
A sub-grain size finite element modelling approach is presented in this paper to investigate variations in fracture mechanics parameters for irregular crack paths. The results can be used when modelling intergranular and transgranular crack growth where creep and fatigue are the dominant failure mechanisms and their crack paths are irregular. A novel method for sub-grain scale finite element mesh consisting of multiple elements encased in $\sim 50-150 \mu \mathrm{m}$ sized grains has been developed and implemented in a compact tension, $\mathrm{C}(\mathrm{T})$, mesh structure. The replicated shapes and dimensions were derived from an isotropic metallic grain structure using representative random sized grain shapes repeated in sequence ahead of the crack tip. In this way the effects of crack tip angle ahead of the main crack path can be considered in a more realistic manner. A comprehensive sensitivity analysis has been performed for elastic and elastic-plastic materials using ABAQUS and the stress distributions, the stress intensity factor and the $J$-integral have been evaluated for irregular crack paths and compared to those of obtained from analytical solutions. To examine the local and macroscopic crack path effects on fracture mechanics parameters, a few extreme cases with various crack-tip angles have been modelled by keeping the macroscopic crack path parallel to the axis of symmetry. The numerical solutions from these granular mesh structures have been found to be in relatively good agreements with analytical solutions.
\end{abstract}

Keywords: intergranular, transgranular, crack path, grain, microstructure

\section{Nomenclature}

$\begin{array}{ll}a & \text { Crack length } \\ B & \text { Specimen thickness } \\ B_{n} & \text { Net specimen thickness between side-grooves } \\ c & \text { Uncracked ligament } \\ E & \text { Elastic (Young's) modulus } \\ E^{\prime} & \text { Effective elastic modulus ( = E for plane stress and } E /\left(1-v^{2}\right) \text { for plane strain) } \\ H & \text { Geometry function in displacement approach for estimation of } J \text {-integral } \\ J & \text { Fracture mechanics parameter for plastically deforming material }\end{array}$

*Corresponding author: ali.mehmanparast@imperial.ac.uk 


\begin{tabular}{|c|c|}
\hline$J_{e}$ & Elastic component of $J$-integral \\
\hline$J_{p}$ & Plastic component of $J$-integral \\
\hline$K, K_{I}$ & Stress intensity factor, Mode-I stress intensity factor \\
\hline$N$ & Plastic hardening exponent \\
\hline$P$ & Load \\
\hline$P_{p 0}$ & Plastic normalising load \\
\hline$q$ & Crack growth direction \\
\hline$r$ & Radial distance ahead of the crack tip \\
\hline$W$ & Specimen width \\
\hline$W_{s}$ & Strain energy density \\
\hline$Y$ & Shape function \\
\hline$\theta$ & Crack tip angle \\
\hline$\eta$ & Geometry function in displacement approach for $J$-integral estimation \\
\hline$\Delta_{p}$ & Plastic displacement \\
\hline$\sigma$ & Nominal stress \\
\hline$\sigma_{y y}$ & Stress distribution in $y$ direction ahead of the crack tip for an elastically deforming material \\
\hline$\sigma_{Y}$ & Yield stress \\
\hline$\sigma_{0.2}$ & $0.2 \%$ proof stress \\
\hline$\sigma_{p 0}$ & Normalising stress in Ramberg-Osgood material model \\
\hline$\sigma_{\text {ref }}$ & Reference stress \\
\hline$\sigma_{e}$ & von Mises equivalent stress \\
\hline
\end{tabular}

\section{Introduction}

A significant number of high temperature components undergo creep or creep/fatigue loading conditions. Assessments are normally required to evaluate the safety of either real or postulated defects in plant components. In addition, there is a need to assess irregular defects in plant components in a sufficiently accurate manner. Defect assessments in these components need laboratory crack growth data to estimate the remaining life [1-3]. Fracture toughness, fatigue and creep crack growth $(\mathrm{CCG})$ tests are usually performed on the compact tension, $\mathrm{C}(\mathrm{T})$, fracture specimen geometry. The specimens are often side grooved to promote crack growth along the plane of the initial starter crack. However, due to the intergranular nature of CCG process, small deviations in the crack path are observed in test specimens (see Figure 1).

Crack paths are often considered straight in macroscopic scale when evaluating the fracture mechanics parameters such as $K$ and $J$ for the case of elastic and elastic-plastic materials, respectively. However, realistic solutions will be provided when the crack deviations above and below the symmetry line are taken into account. The influence of crack tip angle on fracture mechanics parameters $K$ and $J$ is investigated in this paper by extending the crack using defined increments is such a way that intergranular deviations are modelled whereas the macroscopic 
crack path remains straight. The numerical results from these studies on the straight and deviating crack paths are validated through comparisons to the existing analytical solutions for macroscopically straight crack paths.

The level of accuracy and scatter in the data from experimental testing and numerical modelling helps to determine the confidence of the life assessments calculations. As a first step, it is important to compare the numerical solutions of the elastic $K$ and plastic $J$ parameters to the available analytical solutions, by implementing regular and irregular crack paths in mesh structures. For this purpose a novel method of meshing which replicates a polycrystalline grain structure is developed. This would allow the development of simulation methods where damage can grow and coalesce on grain boundaries to form micro-crack and large cracks in an irregular pattern similar to a real case shown in Figure 1. The finite element (FE) method used as a tool to examine fracture mechanics problems at a range of length scales is ideal for this purpose.

Usually it is recommended that a focused mesh at the crack tip should be employed to evaluate fracture mechanics parameters (see e.g. [4]). However this approach is limited to stationary and straight crack tip which does not cater for crack growth modelling unless computationally intensive remeshing techniques are employed. This is unrealistic and therefore methods need to be examined to evaluate the fracture mechanics parameters $J$ and $K$, for growing cracks which are irregular in shape. These methods require explicit modelling of the grain structure to simulate realistic intergranular crack paths. In this paper a number of crack tip angles have been examined and the elastic-plastic energy release rates for crack extensions along different directions are investigated and verified through comparisons with analytical and straight crack path solutions.

\section{Elastic-Plastic Deformation Models}

The Ramberg-Osgood material model is widely used to describe the stress-strain behaviour of strain hardening materials. In non-dimensional, uniaxial form it may be written as

$$
\varepsilon / \varepsilon_{p 0}=\sigma / \sigma_{p 0}+\alpha\left(\sigma / \sigma_{p 0}\right)^{N}
$$

where $\sigma$ and $\varepsilon$ are the stress and strain, respectively, $\alpha$ is a material constant and $\sigma_{p 0}$ and $\varepsilon_{p 0}$ are the normalising (plastic) stress and strain. Note that there is no precise yield stress in this material law, but the non-linear (powerlaw) response typically dominates when $\sigma>\sigma_{p 0}$ and $\sigma_{p 0}$ is often taken to be the $0.2 \%$ proof stress of the material. For multiaxial loading conditions, Eqn (1) may still be applied by employing the von Mises equivalent stress, $\sigma_{e}$, in calculations.

\section{Estimation Methods For Fracture Mechanics Parameters}

In this section an outline of the methods used to derive appropriate fracture mechanics parameters for laboratory specimens is presented.

\subsection{Linear Elastic K Field Solution}

For an infinitely sharp crack tip under linear elastic conditions, the crack tip stress field is described by the stress intensity factor, $K$, according to the relationship [5] 


$$
\sigma_{i j}=\frac{K}{\sqrt{2 \pi r}} f_{i j}(\theta)
$$

where $r$ is the radial distance from the crack tip at an arbitrary crack tip angle, $\theta$. The stresses in the loading direction can be found by defining

$$
f_{y y}=\cos \left(\frac{\theta}{2}\right) \times\left(1+\sin \left(\frac{\theta}{2}\right) \times \sin \left(\frac{3 \theta}{2}\right)\right)
$$

For a sharp crack in a finite body, the stress intensity factor can be estimated using

$$
K=Y\left(\frac{a}{W}\right) \sigma \sqrt{a}
$$

where $\sigma$ is the applied stress, $Y(a / W)$ is a dimensionless function of the geometry and $a$ is the crack length. For a $\mathrm{C}(\mathrm{T})$ specimen the nominal applied stress can be defined as [6]

$$
\sigma=\frac{P}{\sqrt{B B_{n}} W}
$$

where $W$ is the specimen width, $B$ is thickness, $B_{n}$ is net thickness between the side grooves and $P$ is the applied load.

\subsection{J-Definition and HRR Stress Distribution Field}

For a non-linear power law hardening material, the stress distribution field near the crack tip can be defined in terms of $J$-integral by HRR equation (Hutchinson [7, 8] and Rosengren \& Rice [9])

$$
\frac{\sigma_{i j}}{\sigma_{p o}}=\left[\frac{J}{\alpha \varepsilon_{p 0} \sigma_{p o} I_{N} r}\right]^{\frac{1}{N+1}} \tilde{\sigma}_{i j}(\theta ; N)
$$

where $\sigma_{i j}$ is the stress tensor, $r$ is the radial distance from the crack tip and $\tilde{\sigma}_{i j}$ is a non-dimensional function of crack tip angle $\theta$ and plastic deformation stress exponent $N$, values of which are provided in [10]. In Eqn (6), the material parameters $\alpha, \varepsilon_{p 0}$ and $\sigma_{p 0}$ are as defined in the Ramberg-Osgood model (Eqn (1)) and $I_{N}$ is a dimensionless constant which can be estimated using [11]

$$
\begin{aligned}
& \text { For plane stress: } I_{N}=7.2 \sqrt{0.12+1 / N}-2.9 / N \\
& \text { For plane strain: } I_{N}=10.3 \sqrt{0.13+1 / N}-4.6 / N
\end{aligned}
$$

The non-linear elastic crack tip parameter, $J$, may be evaluated from a line integral along a contour surrounding a crack tip [12]. An anticlockwise contour, $\Gamma$, is defined such that

$$
J=\int_{\Gamma} W_{s} d x_{2}-T_{i}\left(\frac{\partial u_{i}}{\partial x_{1}}\right) d s
$$

where $T_{i}$ is the traction vector, $u_{i}$ is the displacement vector, $s$ is the arc length along $\Gamma$ and $W_{s}$ is the strain energy density which can be expressed as

$$
W_{s}=\int_{0}^{\varepsilon_{i j}} \sigma_{i j} d \varepsilon_{i j}
$$


with $\sigma_{i j}$ and $\varepsilon_{i j}$, the stress and strain tensors, respectively. Alternatively the value of $J$ may be obtained from the potential energy release rate of a cracked body $[12,13]$. The line integral solution for $J$ is difficult to solve numerically, therefore alternative methods have been proposed to evaluate the $J$ parameter. A brief review of the relevant methods is next described. A detailed description of all the available methods is provided in [14].

\subsection{Load Line Displacement Approach, $J_{L L D}$}

The potential energy of a cracked body may be related to the load, $P$, and the displacement along the load line, $\Delta^{L L D}$. In order to obtain the value of $J$ directly from the load-displacement curve, it is convenient to split $J$ into elastic and plastic parts [15]. The elastic component of $J, J_{e}$, can be calculated using the linear elastic stress intensity factor, $K$, and the effective elastic modulus, $E^{\prime},\left(=E\right.$ for plane stress and $E /\left(1-v^{2}\right)$ for plane strain conditions) by

$$
J_{e}=\frac{K^{2}}{E^{\prime}}
$$

The plastic component of $J, J_{p}$, may be estimated using

$$
J_{p}=\frac{P \Delta_{p}}{B_{n}(W-a)} H \eta
$$

where $\Delta_{p}$ is the plastic load line displacement and $H$ and $\eta$ are geometry dependent parameters which can be defined as $H=N /(N+1)$ and $\eta=2.2$ for a $\mathrm{C}(\mathrm{T})$ specimen [16].

\subsection{EPRI Estimation Method, $J_{E P R I}$}

Under small scale yielding (SSY) conditions, the elastic component of $J$ may be estimated from $K$ evaluated at the effective crack length, $a_{e}$, and the effective elastic modulus. The plastic component of $J$ may also be given by the EPRI solutions [17]. Therefore, an estimate of the total value of $J$ using the EPRI approach, here denoted $J_{E P R I}$, is given by the sum of the SSY and plastic components as

$$
J_{E P R I}=\frac{K^{2}\left(a_{e}\right)}{E^{\prime}}+\alpha \varepsilon_{p 0} \sigma_{p 0} c h_{1}\left(\frac{a}{W} ; N\right)\left[P / P_{p 0}\right]^{N+1}
$$

where $h_{l}$ is a dimensionless function of normalised crack length and hardening exponent, $N$, and $c$ is the characteristic length scale for the geometry (e.g. uncracked ligament). In Eqn (13), $P_{p 0}$ is the plastic normalising load which is given by

$$
\begin{array}{ll}
\text { For plane stress } & : P_{p 0}=1.071 \Psi c \sigma_{p 0} \\
\text { For plane strain } & : P_{p 0}=1.455 \Psi c \sigma_{p 0}
\end{array}
$$

where $\Psi$ is defined as

$$
\Psi=\sqrt{\left[(2 a / c)^{2}+2(2 a / c)+2\right]}-[(2 a / c)+1]
$$

Solutions of $h_{l}$ have been obtained numerically and tabulated in [17].

\subsection{Domain Integral and VCE Method}

The calculation of the $J$-integral using the line integral approach (see Eqn (9)) is unfavourable in FE models. Area and volume integrals are therefore implemented in numerical codes, for 2D and 3D analyses, respectively. For an 
arbitrary crack path the ' $J$-vector' is introduced, the first component of which is identical to the $J$-integral and corresponds to the case of a straight crack along the $x_{l}$ axis (see Figure 2).

A virtual crack extension (VCE) technique, was introduced by Parks and Hellen [18, 19] and improved by deLorenzi $[20,21]$ to calculate the $J$-vector. In this approach, the crack tip is 'virtually' translated by small crack extension in a given direction and $J$ is defined as an energy release rate for a small fictitious crack extension. The domain integral approach which was proposed by Shih [22, 23] is used in the finite element code ABAQUS [24] and is similar to the VCE method. In the absence of body forces, thermal strains and crack face tractions, the $J$-vector for a linear or nonlinear elastic material under quasistatic conditions can be written as

$$
J=\int_{A^{*}}\left(\sigma_{i j} \frac{\partial u_{j}}{\partial x_{1}}-w \delta_{1 i}\right) \frac{\partial q}{\partial x_{i}} d A
$$

where $A^{*}$ is the area enclosed by $\Gamma^{*}=\Gamma_{1}+\Gamma_{+}+\Gamma_{-}-\Gamma_{0}$ and $\Gamma_{1}, \Gamma_{+}, \Gamma_{-}, \Gamma_{0}$ are outer contour, upper crack face, lower crack face and inner contour, respectively (see Figure 2). In Eqn (16), $q$ is the normalised virtual displacement, which may be associated with an angle $\theta$, and thus is generally defined in the FE codes as the $q$-vector.

Several contour integral evaluations are possible at each location along a crack. In a finite element model each evaluation can be thought of as the virtual motion of a block of material surrounding the crack tip (in two dimensions-2D) or surrounding each node along the crack line (in three dimensions-3D). Each block is defined by contours, where each contour is a ring of elements completely surrounding the crack tip or the nodes along the crack line from one crack face to the opposite crack face. These rings of elements are defined recursively to surround all previous contours.

\section{FE Model Development}

\subsection{Mesh Design and Specimen Geometry}

In this paper the grain structure of a polycrystalline material has been incorporated into a finite element model. A number of approaches have previously been employed to model the grain structure including idealistic hexagonal grains (see e.g. [25-27]) and more realistic grain structures can be produced using the mathematical formulation 'Voronoi tessellation' (see e.g. [28]). A simpler approach has been employed in this work to generate a realistic grain structured mesh. Based on a microstuructural image of Type $316 \mathrm{H}$ stainless steel, a unit cell has been developed in ABAQUS (see Figure 3(a)). The individual grains have been defined by partitioning inside the unit cell. A linear pattern has been used to expand the grain structured unit cell in $x$ and $y$ directions. For clarity, a grain has been highlighted in Figure 3 to indicate the repetitive cell structure. The boundaries of the unit cell are designed in a way such that it may be rotated by $90^{\circ}, 180^{\circ}$ and $270^{\circ}$ and fitted into the combined strucurure. The grain size ranges between around $50-150 \mu \mathrm{m}$, which is typical for $316 \mathrm{H}$ stainless steel material. Although there is no physical grain boundary region in reality, grain boundary elements have been defined to accommodate intergranular damage in future work. Having examined different values of thickness for grain boundaries, the optimum size found to prevent element distortion was $1 \mu \mathrm{m}$. Different material properties may be assigned to various grains and grain boundaries, however, for simplicity uniform properties have been used here. 
The unit cell grain mesh structure has been integrated into the crack tip region of a $2 \mathrm{D}$ model of a $\mathrm{C}(\mathrm{T})$ geometry, as illustrated in Figure 4. In accordance with [1], standard sized C(T) specimen has been modelled of width, $W=50 \mathrm{~mm}$ and thickness to with ratio $B / W=0.5$. The crack is developed from a notch of root radius 0.25 $\mathrm{mm}$, which represents an electro discharge machined (EDM) notch, located at a distance of $20 \mathrm{~mm}$ from the load line. A refined mesh has been employed along the crack tip zone, which is progressively coarsened away from the crack front. Two dimensional plane stress (PS) and plane strain (PE) continuum four noded reduced integration point elements (type CPE4R and CPS4R) have been employed to enable the evaluation of contour integrals. The minimum element size within the grain structured region close to the crack tip is $5 \mu \mathrm{m}$ in this mesh.

The material properties employed are detailed in Table 1. A range of crack lengths and applied loads have been examined under both plane stress and plane strain conditions, as detailed in Table 2. The ratio of the reference stress, $\sigma_{r e f}$, to the yield stress, $\sigma_{Y}$, often taken to be the $0.2 \%$ proof stress of a material, provides an indication of the levels of plasticity in the specimens and can be defined as [11]

$$
\frac{\sigma_{r e f}}{\sigma_{Y}}=\frac{P}{P_{L C}}
$$

where $P$ is the applied load and $P_{L C}$ is the plastic collapse load, solutions of which are provided in [11]. The normalised reference stress ratios for each load and crack length considered in this work are provided in Table 2.

Transgranular crack propagation has been represented by effectively removing elements from the relevant grains directly ahead of the initial EDM notch, whereas intergranular crack growth has been simulated by removing the appropriate grain boundary elements. Note that for the case of intergranular crack growth, the crack deviates somewhat from (above and below) the specimen's centre line.

\subsection{Contour Integral Evaluation}

The focused mesh design which is conventionally used to evaluate contour integrals cannot be employed in the granular mesh design. Therefore, methods to enable contour integral evaluation in this mesh structure have been examined and validated against alternative analytical solutions. To perform a contour integral analysis in ABAQUS, the crack front, the crack tip or crack line, and the crack extension direction must be defined. The crack front and crack tip can be chosen to be at the same node. It is generally known that the results from the first few contours around the crack tip may be inaccurate. Therefore, if the crack front is defined as a larger area surrounding the crack, the contour values are expected to converge quicker and the path independency being improved [24]. When the crack tip angle deviates from the normal to the loading direction, errors will increase in the $J$-contour analysis. However, as long as the main crack is normal to the loading direction and the number of contours is extended beyond the crack tip, the results should converge towards a straight crack.

To evaluate the contour integral around a non-planar intergranular crack, the first contour may be defined at some distance away from the crack tip, to improve the accuracy of the solutions obtained. Examples of the crack front defined for the case of transgranular and intergranular cracks are illustrated in Figure 5(a) and Figure 6(a), respectively. A total number of 50 contours have been used in this analysis, which extend to a distance of approximately $250 \mu \mathrm{m}$ ahead of the crack tip. The 25th and 50th contours are illustrated in Figure 5(b,c) and Figure $6(b, c)$ for straight and deviating cracks, respectively. 
When analysing the deviating intergranular path, three crack tip locations have been considered, as shown in Figure 7(a)-(c). Five different combinations of crack tip locations and virtual crack extensions have been examined, as identified in Figure 8. For Cases 1, 2 and 3 the virtual crack growth direction, $q$, is considered to move along the plane of the initial notch tip i.e. $q=(1,0)$, which corresponds to the general macroscopic crack growth direction. Alternatively, for the cases where the crack tip is at an angle, as seen in Figure 7 (b) and (c), the analysis has also been performed by assuming the virtual crack extension, $q$, to be in the direction defined by the grain boundary (Cases 4 and 5 in Figure 8).

\section{Results and Discussion}

A finite element mesh design has been developed that will enable the intergranular creep crack growth and transgranular fatigue crack growth to be examined and related to fracture mechanics parameters. The $K$ and $J$ contour integrals have been evaluated for both a deviating (intergranular) and straight (transgranular) crack path and verified against analytical solutions.

The elastic values of the normalised $J$ parameter, $J_{e} /(\sigma a)$, evaluated at each of the fifty contours in a straight (transgranular) and deviating (intergranular) crack of normalised length $a / W=0.44$ for an applied load of $30 \mathrm{kN}$, and assuming plane stress conditions, for example, are shown in Figure 9. Similarly, the total (elastic + plastic components) of the $J$ contour integral values normalised by the yield stress and the crack length, $J /\left(\sigma_{Y} a\right)$, are shown in Figure 10, for a normalised reference stress value $\sigma_{\text {ref }} / \sigma_{Y}=1.6$. For the examples shown in these figures Cases 1 and 2, identified in Figure 8, have been shown where the $q$-vector was defined as (1,0) i.e. along a macroscopically straight crack path. Also shown in Figure 9 and Figure 10 are the analytical solutions for the elastic and total values of $J$ (see Eqns (11) and (13)), respectively.

As can be seen in Figure 9 and Figure 10, good agreement is found between the elastic and total values of $J$ for the case of a straight crack, except for the first few contours as expected. However, for the case of a deviating crack under elastic conditions, it is observed in Figure 9 that a large number of contours, which are sufficiently remote from the crack tip region, are required in order to obtain a value close to the analytical solution. As shown in Figure 6 when the number of contours increases, a wider contour region forms ahead of the crack tip. When the contours get sufficiently far away from the crack tip region, the effect of the deviating crack path becomes less significant and thus the expected contour values are obtained. It is therefore apparent that the meandering crack face has a significant effect on the path dependency of the elastic component of the $J$ contour integral. The influence of the crack tip region may be accommodated for, by evaluating the contour integral at a sufficient distance from the crack tip. For the elastic-plastic case shown in Figure 10, the deviating crack appears to have two distinct values of the $J$ parameter close to the crack tip (contours 1-18) and further from the crack tip (contour numbers > 22). Unlike the elastic case (Figure 9), the values for the remote contours fall slightly below that of the analytical solution (1.9\% error for Case 1 and $5.1 \%$ error for Case 2), but are apparently path independent. Note that significant crack tip blunting may take place under plastic deformation conditions, which may influence the results obtained.

Good agreement has been found between the elastic crack tip stress field evaluated from the finite element analysis and the $K$ field solution (eqn (2) ), as shown for example in Figure 11 where the normalised stress parallel 
to the loading direction, $\sigma_{y y} / \sigma$, is plotted against normalised crack tip distance for the case of transgranular and intergranular cracks at $a / W=0.44$. The equivalent crack tip stress fields obtained from each of the cases considered are also compared to the HRR field solution in Figure 12. Due to the mesh geometry, the node from which the stress values is being extracted may be located at some arbitrary crack tip angle, $\theta$, thus the HRR field solutions are shown for $\theta=0^{\circ}$ and $\theta=20^{\circ}$ for comparison purposes. The normalised equivalent stress calculated is in relatively good agreement with the HRR field solution under plane stress conditions for all cases considered. Under plane strain conditions the evaluated stress falls between the HRR field solution at $\theta=0^{\circ}$ and $\theta=20^{\circ}$, for the straight crack and also the deviating crack with $r / a>0.01$. Due to the localised crack tip constraint effects close to the deviating crack tip, the stress calculated is found to be higher than that of the straight crack and HRR field solution under plane strain conditions.

A comparison of the contour integral values obtained for the range of crack lengths and stress states examined are next presented. The stress intensity factor under Mode-I dominant conditions is first presented in Figure 13, where excellent agreement is found between the FE and analytical solution for the range of crack length examined for the effectively straight (transgranular) crack. Similarly, the elastic components of the $J$ parameter, presented in the normalised form, are in agreement with the analytical solutions under both plane stress and plane strain conditions, as shown in Figure 14.

The elastic value of the $J$ parameter is shown in Figure 15 for all the deviating (intergranular) crack cases examined. Case $1-3$, where the $q=(1,0)$, are in relatively good agreement with the analytical solutions, with Cases 1 and 2 being almost identical. However, for Cases 4-5, where the crack is assumed to grow along the grain boundary direction (see Figure 8), the values of $J$ predicted are less than that of Cases 1 and 2, and the analytical solution. Hence, the energy release rate for crack path deviation along these grain boundaries in Cases 4 and 5 is less than that of a straight fronted crack. This can be attributed to the contribution of shear stresses present at the deviating crack tip, which may increase with crack length. As indicated in Figure 16, the Mode-I component of linear elastic stress intensity factor, $K_{I}$, for Cases 4-5 falls significantly below the values found in Cases 1-3, and below the analytical solution. Note that the $K_{I}$ values for Cases 1-3 are close to the analytical solution. A conservative estimate of $K_{I}$ will therefore be obtained if the straight crack solution is applied to the case of a deviating crack.

The total values of $J$, from an elastic-plastic analysis, have also been evaluated and are compared to the load line displacement and EPRI solutions (Eqns (11)-(13)) for a transgranular and intergranular crack in Figure 17 and Figure 18, respectively. Excellent agreement is found between the FE calculations and analytical solutions for all cases examined for the transgranular crack. For the intergranular crack, as shown in Figure 18, the normalised $J$ values evaluated in the FE analysis for Cases 1-3 (with the $q$-vector defined as $(1,0)$ ) are close to displacement and EPRI estimations under both plane stress and plane strain conditions. The lowest value of $J$ is calculated in the FE analysis when the $q$-vector is defined in a direction parallel to load axis i.e. the crack driving force normal to the loading direction is small suggesting that the crack will not move in that direction, though in reality this is the path that an intergranular crack may take. The LLD displacement measurements obtained from the FE analyses may be considered identical for Cases 1-5, and hence the same values of the $J$ parameter were found from the LLD measurement method. Also included in this figure are the $J$ parameter solutions based on the LLD method for a 
straight crack. The results from the straight and deviating crack are almost identical, indicating that the load line displacement measurements remote from the crack tip are uninfluenced by the small variations in the crack profile. This mesh may therefore be used in future work to examine creep damage and intergranular crack growth effects on the macroscopic response of specimens tested at elevated temperatures.

\section{Conclusions}

A sub-grain scale finite element (FE) fracture mechanics analysis has been presented in this paper. A novel unit cell structure to represent an isotropic material microstructure has been used to allow irregular crack paths being modelled. Methods have been examined to evaluate the fracture mechanics parameters $J$ and $K$ in FE analyses on a compact tension, $\mathrm{C}(\mathrm{T})$, geometry using a mesh appropriate for the evaluation of both intergranular and transgranular crack paths. The finite element model consider irregular grain paths at grain size level whilst the macro crack path is kept parallel to the axis of symmetry. Realistic grain and grain boundary microstructures were modelled in the mesh employed, and straight transgranular and deviating intergranular crack paths considered. Excellent agreement was found between the FE contour integral values and analytical solutions for $K$ and $J$ for the case of a straight fronted transgranular crack. Good agreement was also found between the corresponding crack tip stress fields. Reasonable values of $K$ and $J$ parameters could also be achieved from the FE contour integral values of a deviating crack when the contours selected were adequately far from the crack tip. The elastic and plastic components of the $J$ parameter for a deviating crack were found to be in reasonable agreement with the analytical solutions when the virtual crack growth direction ( $q$-vector) was defined as the macroscopic crack growth direction. However, significant differences were found between the analytical and FE contour integral values of a deviating crack when the crack growth direction was considered to continue at the angle defined by its grain boundary. The results of the $J$ parameter obtained from the straight and deviating crack based on the load line displacement method are almost identical, indicating that the load line displacement measurements remote from the crack tip are uninfluenced by the small variations in the crack front geometry.

\section{References}

1. ASTM, E1457-07: Measurement of Creep Crack Growth Rates in Metals, in Annual Book of ASTM Standards, Vol. 03.01, 2007, ASTM International, pp. 1012-1035.

2. R5, Defect assessment code of practice for high temperature metallic components, British Energy Generation Ltd, 2000.

3. British Standard, BS 7910: Guide on Methods for Assessing the Acceptability of Flaws in Metallic Structures, BSI, London, 1999.

4. O'Dowd, N. P. and Shih, C. F., Family of Crack Tip Fields Characterised by a Triaxiality Parameter-I. Structure of Fields, Journal of the Mechanics and Physics of Solids, 1991, 39(8), pp. 989-1015.

5. Williams, M. L., On the Stress Distribution at the Base of a Stationary Crack, Journal of Applied Mechanics, 1957, 24, pp. 109-114.

6. Tada, H., Paris, P. C. and Irwin, G. R., The Stress Analysis of Cracks Handbook, 1985, Saint Louis: Paris Productions \& (Del Research Corp.).

7. Hutchinson, J. W., Singular Behaviour at the End of a Tensile Crack in a Hardening Material, Journal of the Mechanics and Physics of Solids, 1968, 16(1), pp. 13-31.

8. Hutchinson, J. W., Plastic Stress and Strain Fields at a Crack Tip, Journal of the Mechanics and Physics of Solids, 1968, 16(5), pp. 337-342. 
9. Rice, J. R. and Rosengren, G. F., Plane Strain Deformation Near a Crack Tip in a Power Law Hardening Material, Journal of the Mechanics and Physics of Solids, 1968, 16(1), pp. 1-12.

10. Shih, C. F., Tables of Hutchinson-Rice-Rosengren Singular Field Quantities, MRL E-Providence, Ri, Brown University Technical Report, MRL E-147, June, 1983.

11. Webster, G. A. and Ainsworth, R. A., High Temperature Component Life Assessment, 1st ed, 1994, London: Chapman and Hall.

12. Rice, J. R., A Path Independent Integral and the Approximate Analysis of Strain Concentration by Notches and Cracks, Journal of Applied Mechanics, Transactions of the ASME, 1968, 35, pp. 379-386.

13. Budiansky, E. a. R., J.R.,, Conservation laws and energy release rates, Journal of Applied Mechanics, 1973, Vol 40, pp. pp. 201-203.

14. Anderson, T. L., Fracture Mechanics: Fundamentals and Application, 1991, Boston: CRC Press.

15. Ainsworth, R. A., The Assessment of Defects in Structures of Strain Hardening Material, Engineering Fracture Mechanic, 1984, 19, pp. 633-642.

16. Davies, C. M., Kourmpetis, M., O'Dowd, N. P. and Nikbin, K. M., Experimental Evaluation of the J or $C^{*}$ Parameter for a Range of Cracked Geometries, Journal of ASTM International, 2006, 3(4), DOI: 10.1520/JAI13220.

17. Kumar, V., German, M. D. and Shih, C. F., An Engineering Approach for Elastic-Plastic Fracture Analysis, EPRI, Palo Alto, CA, EPRI Report NP-1931, July, 1981.

18. Parks, D. M., A Stiffness Derivative Finite Element Technique for Determination of Crack Tip Stress Intenisty Factor, International Journal of Fracture Mechanics, 1974, Vol. 10, pp. pp. 487-502.

19. Hellen, T. K., On The Method of Virtual Crack Extensions, International journal for numerical methods in engineering, 1975, Vol 9, pp. pp. 187-207.

20. deLorenzi, H. G., On the Energy Release Rate and the J-Integral of 3-D Crack Configurations, international journal of Fracture, 1982, Vol 19, pp. pp. 183-193.

21. DeLorenzi, H. G., Energy Release Rate Calculations by the Finite Element Method, Engineering fracture mechanics, 1985, Vol 21, pp. pp. 129-143.

22. Shih, C. F., Energy Release Rate Along a Three Dimensional Crack Front in a Thermally Stressed Body, International journal of Fracture, 1986, Vol 30, pp. pp. 79-102.

23. Moran, B. and Shih, C. F., A General Treatment of Crack Tip Contour Integrals, International journal of Fracture, 1987, Vol 35, pp. pp. 295-310.

24. ABAQUS, User Manual. 2008, in Version 6.8, SIMULIA. Hibbit, Karlsson, and Sorensen HKS Inc

25. Onck, P. and van der Giessen, E., Microstructurally-based modelling of intergranular creep fracture using grain elements, Mechanics of Materials, 1997, 26(2), pp. 109-126.

26. van der Giessen, E., Development of final creep failure in polycrystalline aggregates, Acta metallurgica et materialia, 1994, 42(3), pp. 959-73.

27. van der Giessen, E., Effect of random variations in microstructure on the development of final creep failure in polycrystalline aggregates, Modelling and Simulation in Materials Science and Engineering, 1994, 2(3), pp. 721-38.

28. Weyer, S., Automatic finite element meshing of planar Voronoi tessellations, Engineering fracture mechanics, 2002, 69(8), pp. 945-58. 
Tables:

Table 1: $316 \mathrm{H}$ stainless steel material properties at $550^{\circ} \mathrm{C}$

\begin{tabular}{|c|c|}
\hline$v$ & 0.3 \\
\hline$E$ & $140 \mathrm{GPa}$ \\
\hline$N$ & 3 \\
\hline$\alpha$ & 5.79 \\
\hline$\sigma_{p 0}$ & $170 \mathrm{MPa}$ \\
\hline
\end{tabular}

Table 2: The normalised reference stress values at different crack lengths and load levels, under PS and PE conditions

\begin{tabular}{|c|c|c|c|c|c|c|c|c|c|c|c|c|c|c|}
\hline $\boldsymbol{a} / \boldsymbol{W}$ & $\mathbf{0 . 4 4}$ & $\mathbf{0 . 4 6}$ & $\mathbf{0 . 4 8}$ & $\mathbf{0 . 5 0}$ & $\mathbf{0 . 5 2}$ & $\mathbf{0 . 5 4}$ & $\mathbf{0 . 5 6}$ & $\mathbf{0 . 5 8}$ & $\mathbf{0 . 6 0}$ & $\mathbf{0 . 6 2}$ & $\mathbf{0 . 6 4}$ & $\mathbf{0 . 6 6}$ & $\mathbf{0 . 6 8}$ & $\mathbf{0 . 7 0}$ \\
\hline 30kN-PS & 1.22 & 1.33 & 1.45 & 1.59 & 1.74 & 1.92 & 2.12 & 2.36 & 2.63 & 2.95 & 3.32 & 3.77 & 4.30 & 4.95 \\
\hline 30kN-PE & 0.83 & 0.91 & 1.00 & 1.10 & 1.21 & 1.33 & 1.48 & 1.65 & 1.84 & 2.07 & 2.34 & 2.67 & 3.05 & 3.53 \\
\hline $\mathbf{4 0 k N - P S}$ & 1.63 & 1.77 & 1.93 & 2.12 & 2.32 & 2.56 & 2.83 & 3.14 & 3.50 & 3.93 & 4.43 & 5.02 & 5.74 & 6.60 \\
\hline $\mathbf{4 0 k N - P E}$ & 1.11 & 1.22 & 1.33 & 1.46 & 1.61 & 1.78 & 1.97 & 2.20 & 2.46 & 2.77 & 3.13 & 3.56 & 4.07 & 4.70 \\
\hline
\end{tabular}

\section{Figures:}

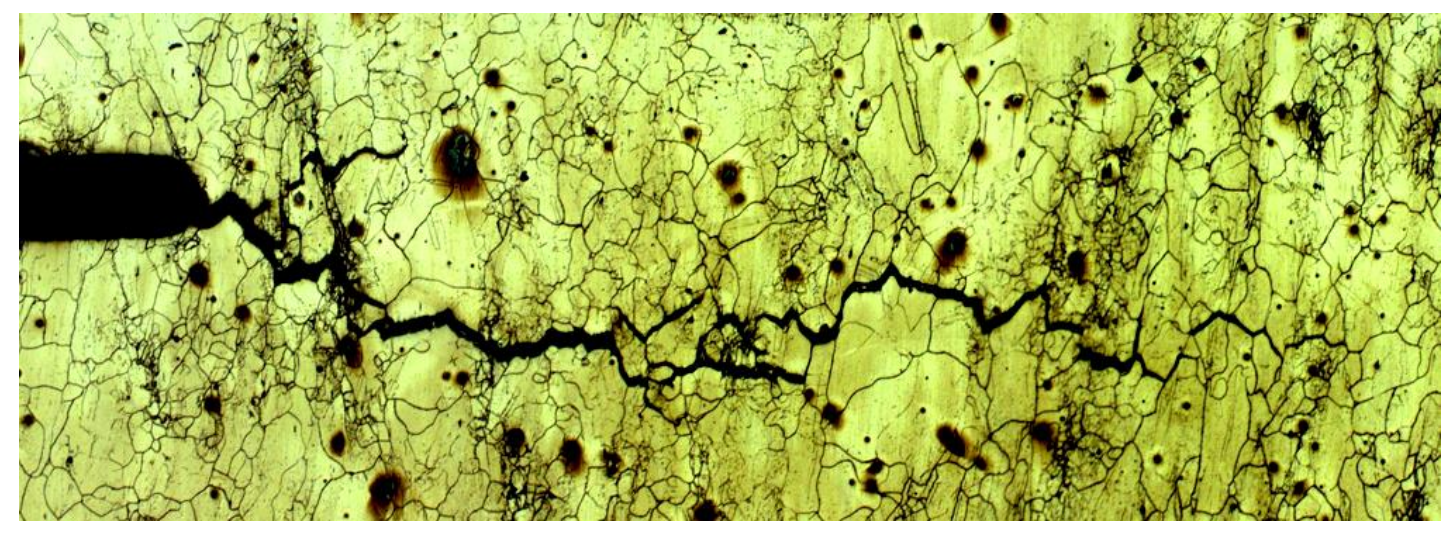

Figure 1: Intergranular creep cracking in 1/2CrMoV. 


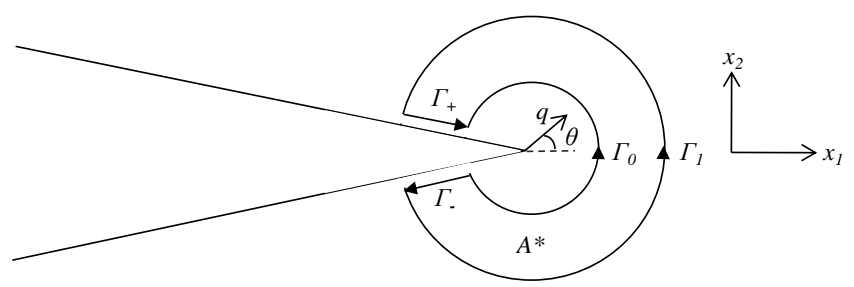

Figure 2: Inner and outer contours forming a closed contour around the crack tip

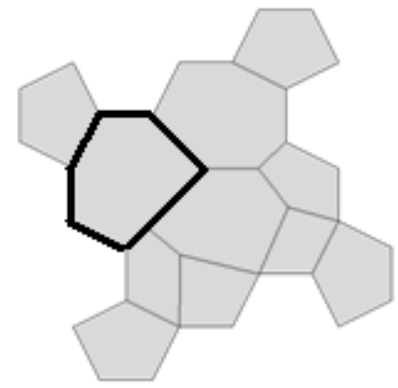

(a)

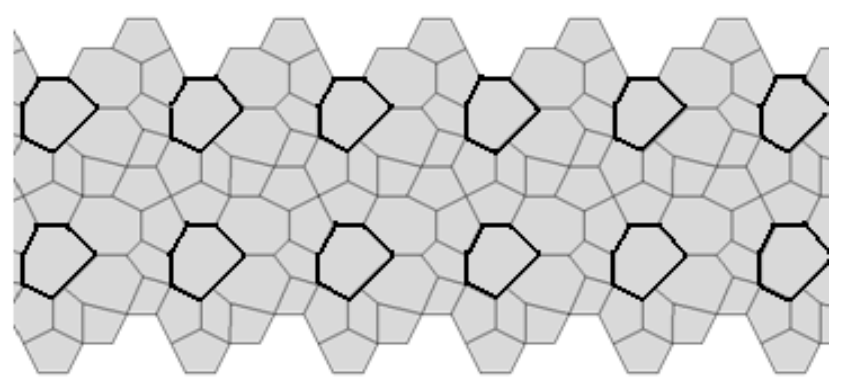

(b)

Figure 3: Illustration of the (a) unit cell of grains and (b) two dimensional linear pattern expansion of unit cells

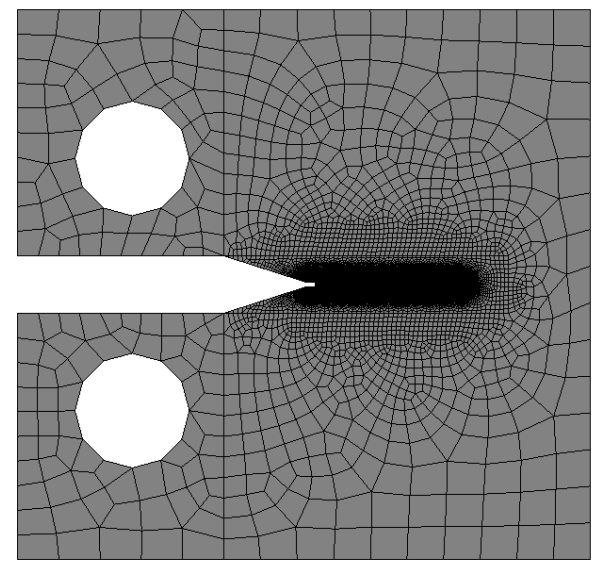

Figure 4: $C(T)$ specimen grain mesh structure 


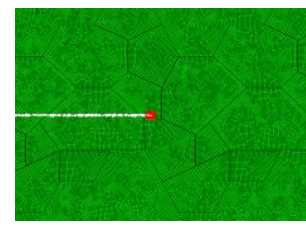

(a)

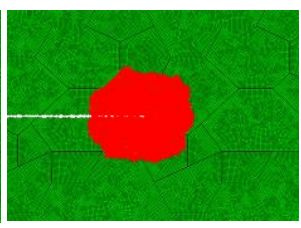

(b)

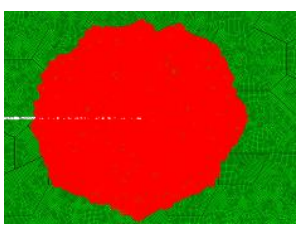

(c)

Figure 5: Illustration of (a)1st (b)25th (c)50th contours around the crack tip for a straight crack

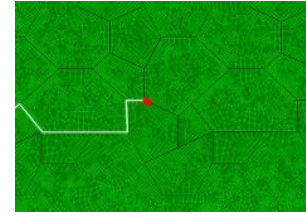

(a)

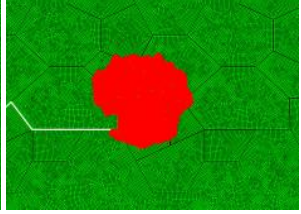

(b)

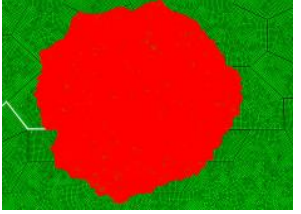

(c)

Figure 6: Illustration of (a)1st (b)25th (c)50th contours around the crack tip for a deviating crack

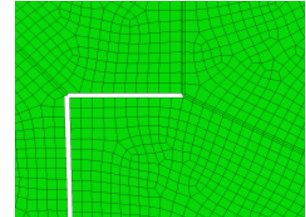

(a)

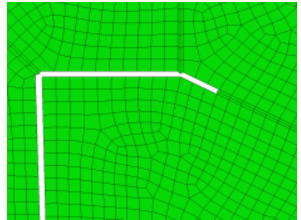

(b)

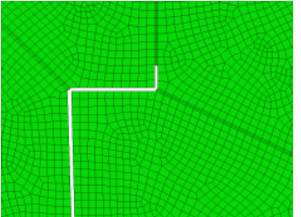

(c)

Figure 7: Examination of different crack tip angles at a triple junction in a deviating crack

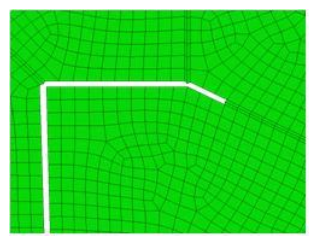

Case 1: $q=(1,0), \theta=0$

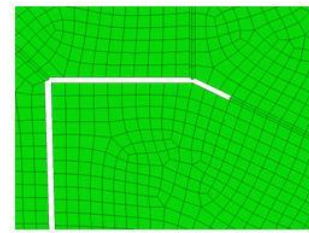

Case 4: $\mathrm{q}=(2,-1), \theta=-30^{\circ}$

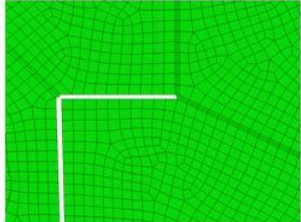

Case 2: $q=(1,0), \theta=0$

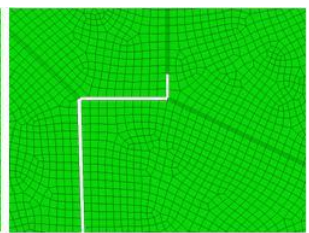

Case 3: $\mathrm{q}=(1,0), \theta=0$

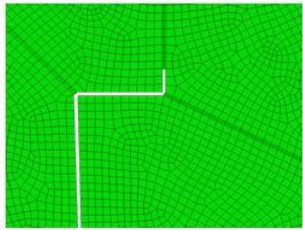

Case 5: $\mathrm{q}=(0,1), \theta=90^{\circ}$

Figure 8: Definition of $q$-vector for possible crack deviation conditions at a triple junction 


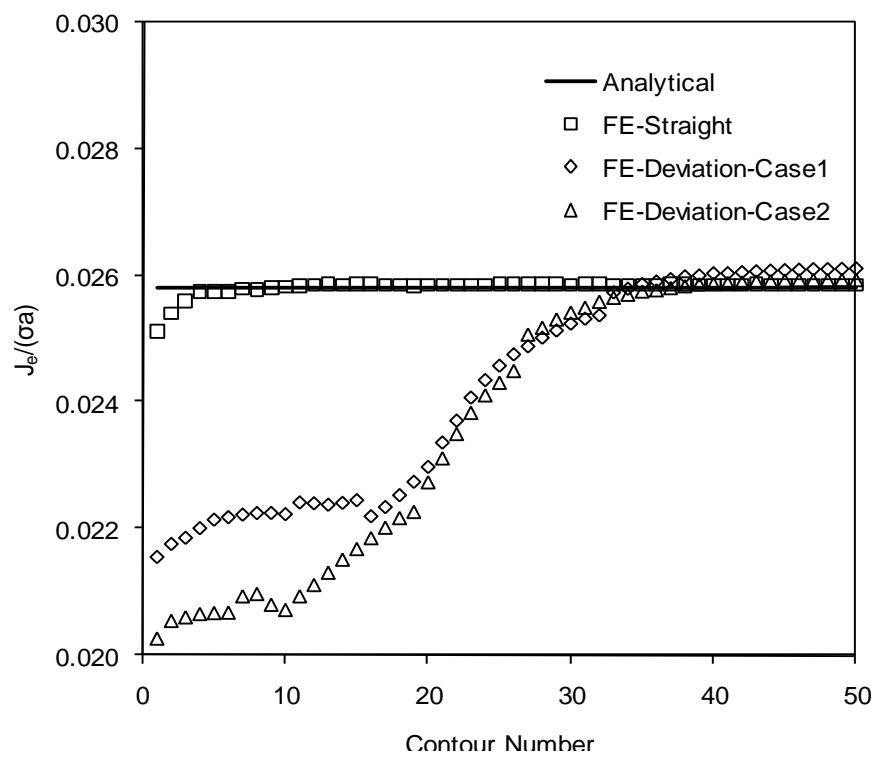

Figure 9: Local and Global values of normalised $J$-elastic for a deviating crack under $30 \mathrm{kN}$ plane stress conditions at $a / W=0.44$

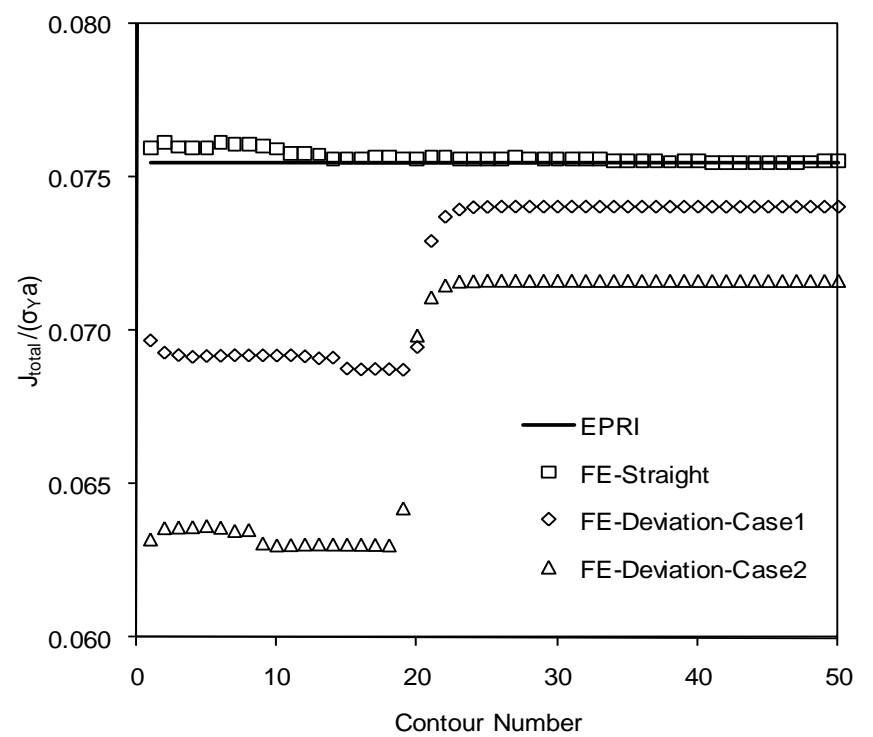

Figure 10: Local and Global values of normalised $J$ for a deviating crack under $40 \mathrm{kN}$ plane stress conditions at $a / W=0.44$ 


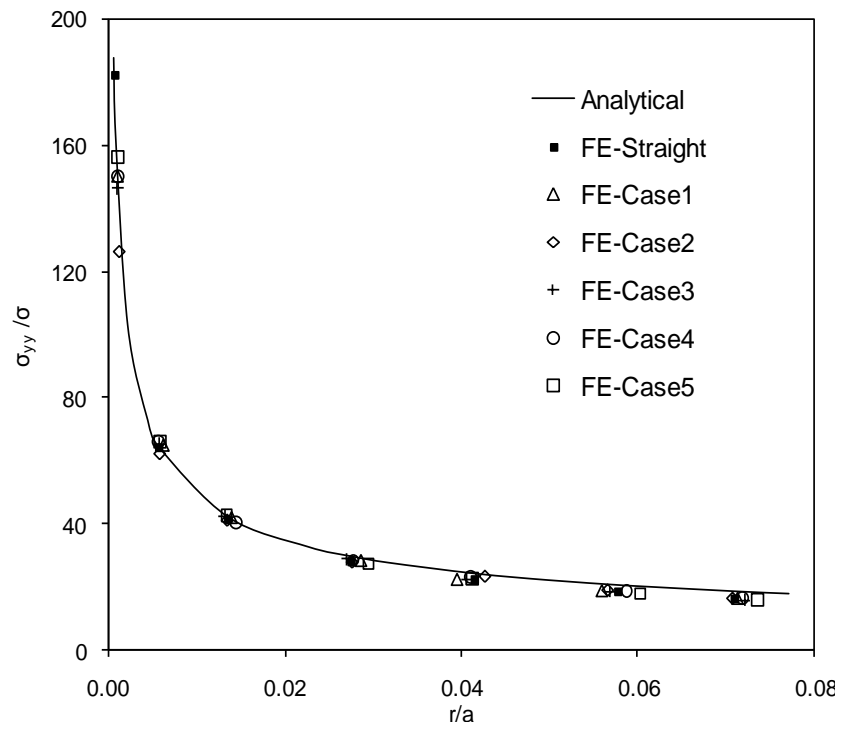

Figure 11: Normalised $K$-field stress distribution ahead of the crack tip in the $y$-direction under $30 \mathrm{kN}$ applied load for a straight and deviating crack at $a / W=0.44$

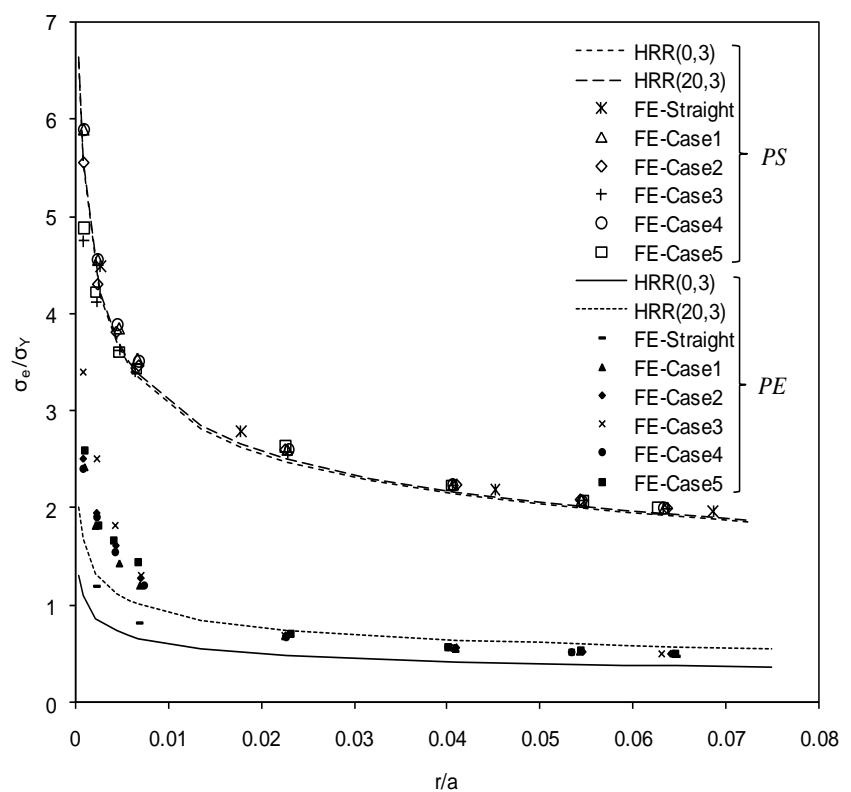

Figure 12-Normalised equivalent stress distribution ahead of the crack tip under $30 \mathrm{kN}$ applied load for a straight and deviating crack at $a / W=0.44$ 


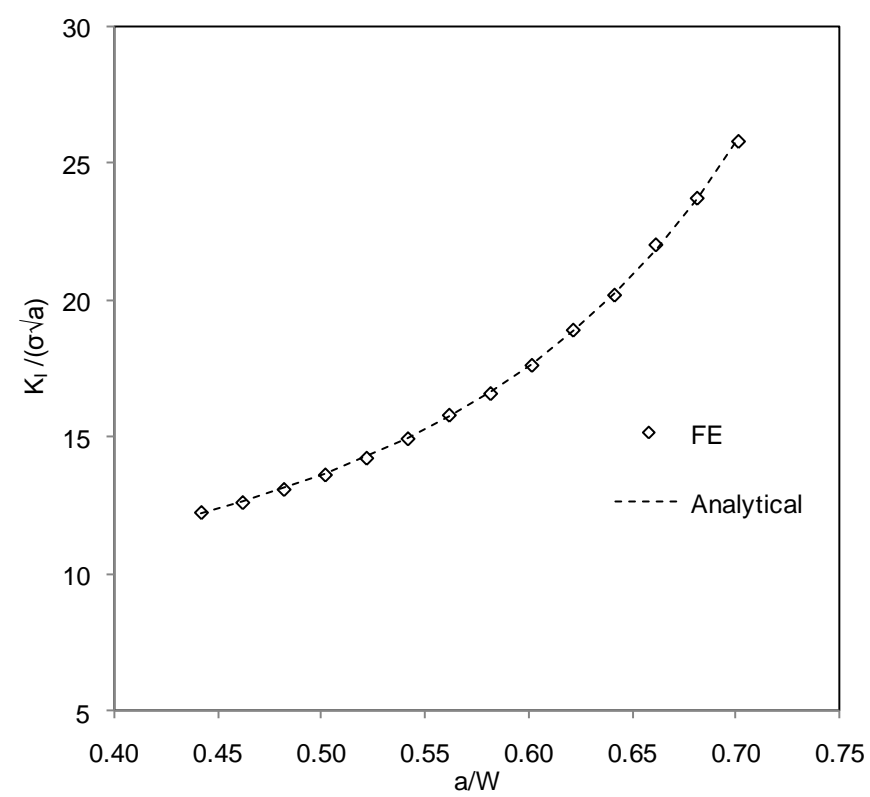

Figure 13: Normalised $K_{I}$ v.s. $a / W$ data for straight crack under $30 \mathrm{kN}$ applied load

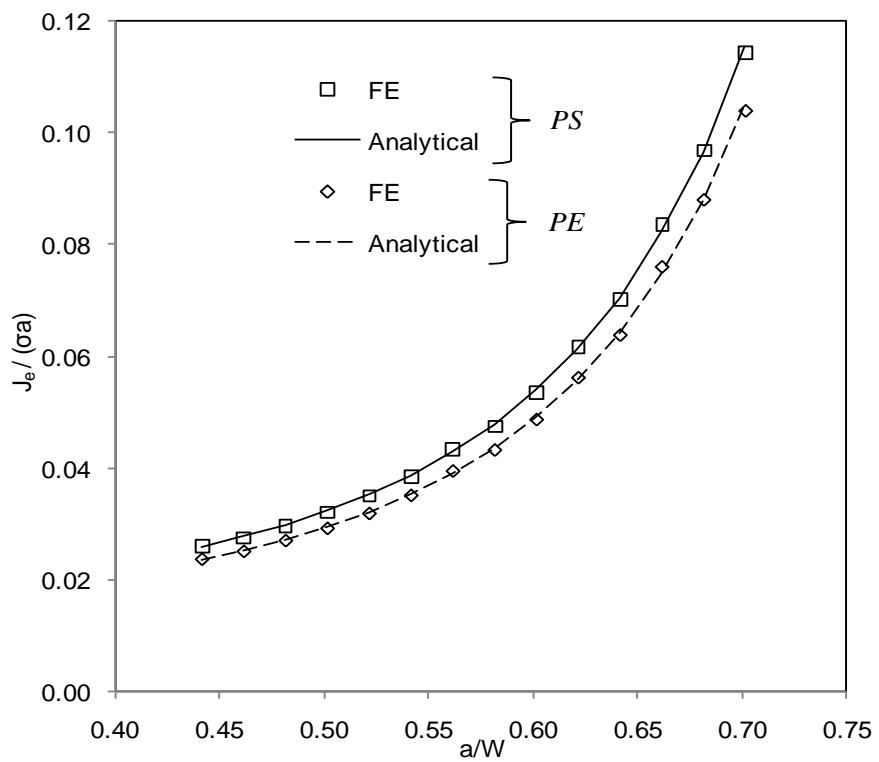

Figure 14: Normalised $J_{e}$ v.s. $a / W$ data for straight crack under $30 \mathrm{kN}$ plane stress and plane strain conditions 


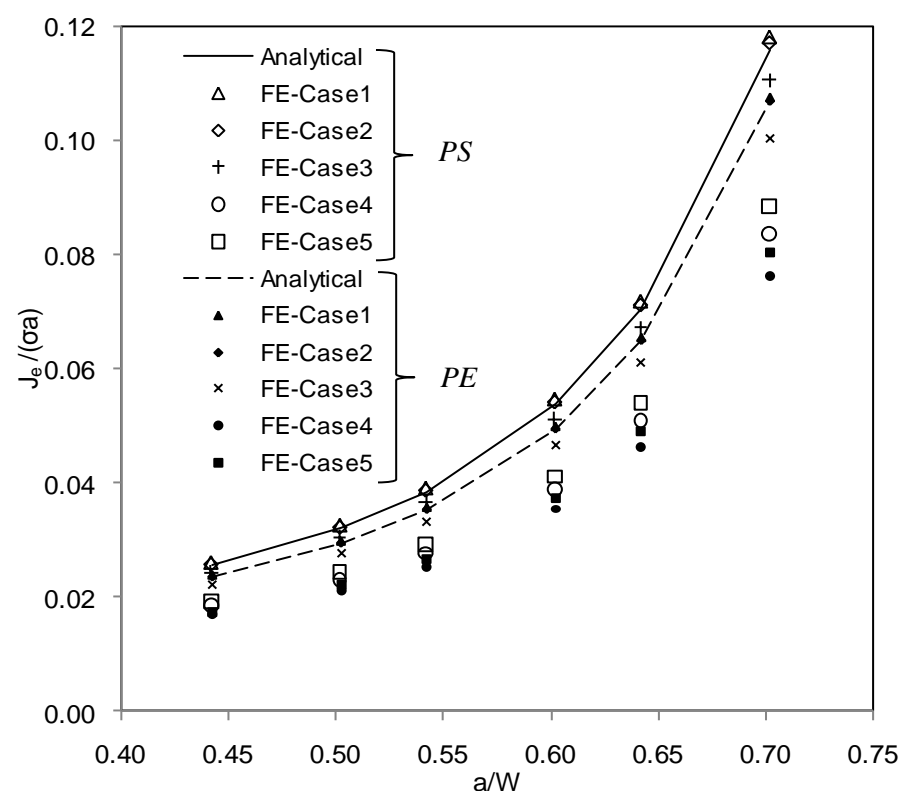

Figure 15: Normalised $J_{e}$ plotted against $a / W$ for a deviating crack under $30 \mathrm{kN}$ plane stress and plane strain conditions

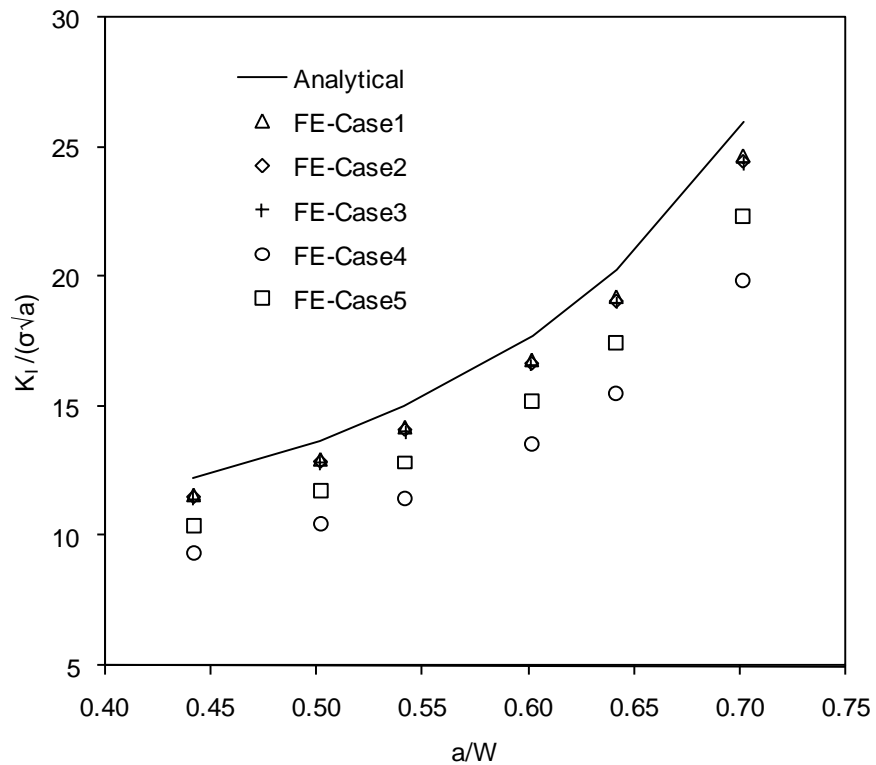

Figure 16: Normalised $K_{I}$ plotted against $a / W$ for a deviating crack under $30 \mathrm{kN}$ applied load 


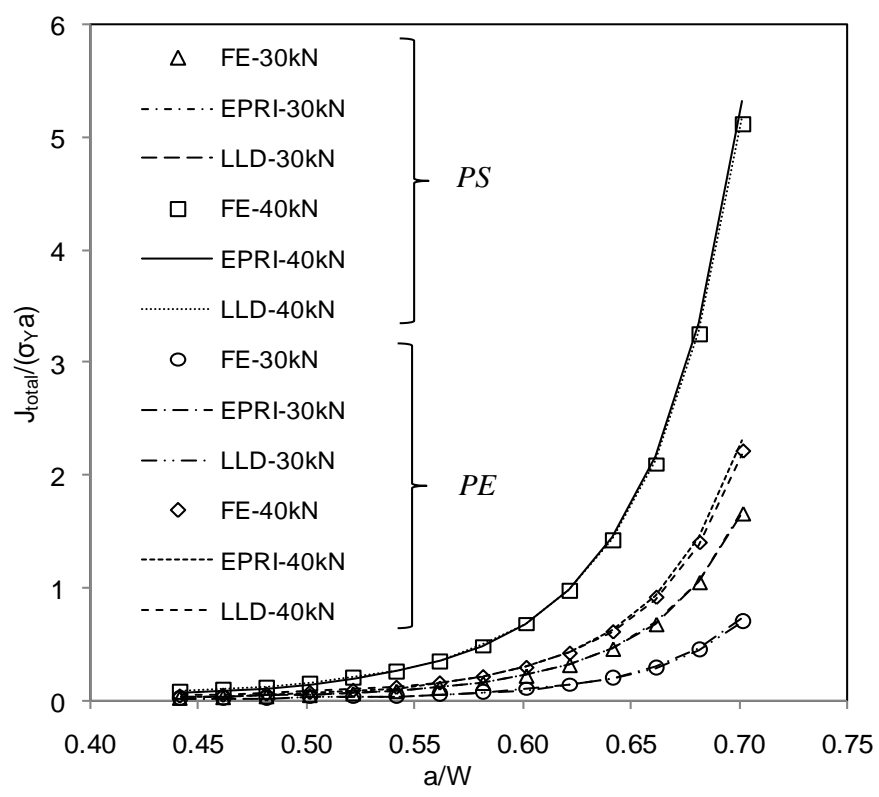

Figure 17: Normalised $J$-total plotted against $a / W$ for a straight crack under $30 \mathrm{kN}$ and $40 \mathrm{kN}$ applied load

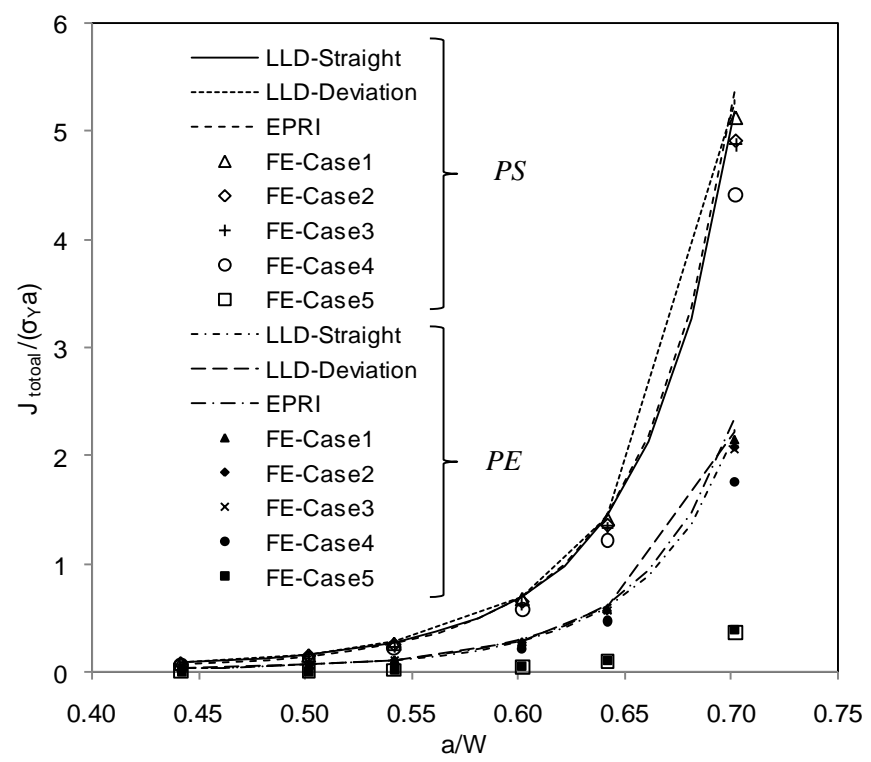

Figure 18: Normalised $J$-total plotted against $a / W$ for a deviating crack under $40 \mathrm{kN}$ applied load 